\title{
Propofol maintains Th17/Treg cell balance and reduces inflammation in rats with traumatic brain injury via the miR-145-3p/NFATc2/NF-кB axis
}

\author{
CAN CUI $^{1 *}$, DENGWEN ZHANG $^{1 *}$, KE SUN $^{1}$, HAIFENG LI $^{1}$, LIQIAN XU $^{1}$, GEN LIN $^{1}$, \\ YUANBO GUO $^{1}$, JIAQI HU ${ }^{1}$, JIEYUAN CHEN ${ }^{1}$, LIDAN NONG ${ }^{1}$, YUJIN CAI ${ }^{1}$, \\ DONGNAN YU ${ }^{1}$, WEI YANG ${ }^{1}$, PENG WANG $^{2}$ and YI SUN ${ }^{1}$ \\ Departments of ${ }^{1}$ Anesthesiology and ${ }^{2}$ Neurosurgery, Guangdong Provincial People's Hospital, \\ Guangdong Academy of Medical Sciences, Guangzhou, Guangdong 510080, P.R. China
}

Received July 29, 2020; Accepted April 2, 2021

DOI: $10.3892 / \mathrm{ijmm} .2021 .4968$

\begin{abstract}
Propofol is a commonly used intravenous anesthetic. The aim of the study was to examine the mechanism of propofol in traumatic brain injury (TBI) by regulating interleukin (IL)-17 activity and maintaining the Th17/Treg balance. A rat model with moderate TBI was established using the weight-drop method. Rats with TBI were regularly injected with propofol and their brain injuries were monitored. The peripheral blood of rats was collected to measure the Th17/Treg ratio. MicroRNA (miR)-145-3p expression was detected in the brain tissues of rats and antagomiR-145-3p was injected into the lateral ventricles of their brains to verify the effect of miR-145-3p on brain injury. The downstream target of miR-145-3p was predicted. The targeting relationship between miR-145-3p and nuclear factor of activated T cells c2 (NFATc2) was confirmed. NFATC2 expression and phosphorylation of $\mathrm{NF}-\kappa \mathrm{B}$ pathway-related proteins were measured. Propofol alleviated brain injury in rats with TBI and maintained the Th17/Treg balance. Propofol upregulated miR-145-3p expression in rat brains, while the inhibition of miR-145-3p reversed the effect of propofol on brain injury. A binding relationship was observed between miR-145-3p and NFATc2. Furthermore,
\end{abstract}

Correspondence to: Dr Yi Sun, Department of Anesthesiology, Guangdong Provincial People's Hospital, Guangdong Academy of Medical Sciences, 96 Dongchuan Road, Guangzhou, Guangdong 510080, P.R. China

E-mail: sunnyuva2014@yahoo.com

Dr Peng Wang, Department of Neurosurgery, Guangdong Provincial People's Hospital, Guangdong Academy of Medical Sciences, 96 Dongchuan Road, Guangzhou, Guangdong 510080, P.R. China E-mail: wangpeng_82@sina.com

*Contributed equally

Key words: propofol, traumatic brain injury, microRNA-145-3p, nuclear factor of activated $\mathrm{T}$ cells $\mathrm{c} 2$, Th17/Treg, NF- $\kappa \mathrm{B}$, inflammation propofol decreased the phosphorylation of p65 and I $\kappa \mathrm{B} \alpha$, and inhibited activation of the $\mathrm{NF}-\kappa \mathrm{B}$ pathway in the brains of rats with TBI. In conclusion, propofol maintained Th17/Treg balance and reduced inflammation in the rats with TBI via the miR-145-3p/NFATc2/NF- $\mathrm{BB}$ axis.

\section{Introduction}

Traumatic brain injury (TBI) is a type of acute brain injury caused by the mechanical energy of external force acting on the head (1). TBI poses an enormous financial and societal burden with high morbidity and mortality (2). Following the damage caused at the time of brain injury (primary injury), various events that occur within minutes to days after the injury can lead to secondary injury (3). The long-term outcomes of patients with TBI can be ameliorated by prompt and effective treatment after trauma (4). Thus, further elucidation of the complicated cellular and molecular events contributing to secondary injury is crucial. Neuroinflammation is reported to be a critical factor inducing secondary brain injury, and suppression of inflammation is a favorable approach to alleviate secondary brain injury and improve prognosis in TBI (5). The inflammatory cascade is activated by the release of pro-inflammatory and anti-inflammatory cytokines (6). Cytokines, such as interleukin (IL)-17, are notably upregulated after TBI, which is concerned with the pathogenesis of TBI (7). An imbalance in Th17/Treg cells is considered a pivotal factor in the progression of the inflammatory response $(8,9)$. Therefore, maintaining the Th17/Treg balance may be an effective approach for managing TBI.

Propofol, a short-acting intravenous anesthetic, has been extensively utilized in the management of craniocerebral injury for induction, maintenance, and sedation during surgery (10). Propofol also reduces the generation of pro-inflammatory cytokines (11) and has been reported to exert a neuroprotective effect on diverse neuronal injuries, such as ischemic stroke and TBI (12). However, the detailed mechanism underlying propofol's protective effect on TBI has not been elucidated.

Emerging evidence has shown that propofol plays an anti-inflammatory and neuroprotective role by regulating microRNAs (miRs) and their downstream targets $(12,13)$. 
miRs are endogenous non-coding RNAs that regulate protein-coding genes post-transcriptionally (14). Dysregulation of miRs has been reported to be involved in the pathological process of TBI (15). For example, Henry et al revealed that the suppression of miR-155 can reduce neuroinflammation and promote functional restoration after TBI in mice (16). In view of this, a TBI model in rats was established to explore the mechanism by which propofol protects against brain injury by modulating miR and its downstream targets. The findings may provide promising therapeutic targets for patients with TBI to attenuate brain injury and improve prognosis.

\section{Materials and methods}

Ethics statement. The study was approved by the Ethics committee of the Guangdong Provincial People's Hospital. All experimental procedures were performed in accordance with the Ethical Guidelines for the Study of Experimental Pain in Conscious Animals.

Establishment of TBI model in rats and grouping (17). Male Sprague Dawley (SD) rats (aged 12-16 weeks, weighing 360-400 g), purchased from the Animal Research Center of Wuhan University [SCXK (Hubei) 2019-0004, Wuhan, Hubei, China] were kept at the experimental site for at least one week prior to surgery. SD rats were raised in separate cages in an animal room at $23-25^{\circ} \mathrm{C}$ and $50-60 \%$ humidity under a 12-h light/dark cycle, and given normal clean feed. The rats had free access to food and water. The padding was replaced every other day to keep the cage clean. The rats were anesthetized via intraperitoneal injection of $1 \%$ pentobarbital (30 mg/kg). The rats were then fixed, shaven, and disinfected, and the scalp was cut to expose the skull. A 5-mm-diameter hole $(2.0 \mathrm{~mm}$ from the posterior dura and $2.0 \mathrm{~mm}$ to the right of the sagittal suture) was drilled in the rats' skulls, and the dura was exposed. Moderate TBI was caused in the rats by a 40-g weight-drop from a height of $25 \mathrm{~cm}$. The rats in the sham-operation group were operated on without a weight-drop. After the weight-drop, the scalps of the rats were sutured, and the rats recovered from anesthesia. All the operations were performed using aseptic techniques. TBI was confirmed by the observation of limb convulsions, transient apnea, and unconsciousness in rats.

The rats with TBI were assigned to five groups $(n=18$ in each group) as follows: Sham group, TBI group, TBI + propofol group $(\mathrm{TBI}+\mathrm{P}), \mathrm{TBI}+$ fat emulsion group $(\mathrm{TBI}+\mathrm{FB})$, $\mathrm{TBI}+$ propofol + antigomiR-145-3p group (TBI+P+ant-145), and $\mathrm{TBI}+$ propofol + antigomiR NC group (TBI+P+ant-NC). In the $\mathrm{TBI}+\mathrm{P}$ group, the rats were intravenously injected with propofol over $5 \mathrm{~min}(12.5 \mathrm{mg} / \mathrm{kg}) 1 \mathrm{~h}$ after TBI and then infused intravenously at $40 \mathrm{mg} / \mathrm{kg} / \mathrm{h}$ over $2 \mathrm{~h}$. Clinical doses of propofol were used based on preliminary experiments and pharmacological calculations. Fat emulsion, which was injected intravenously over $5 \mathrm{~min} 1 \mathrm{~h}$ after TBI and then continuously infused for $2 \mathrm{~h}$ at the same dosage and infusion rate as that of propofol in the TBI+P group, was used as the solvent control for propofol. Then, $1 \mathrm{~h}$ after establishing TBI, $0.5 \mathrm{nmol}$ of ant-NC (Invitrogen, Inc.) was administered into the brains of rats by intracerebroventricular injection and repeated every $1 \mathrm{~h}$ for three total injections.
Blood $(1.5 \mathrm{ml})$ was collected from the rats' orbits $24 \mathrm{~h}$ after establishing TBI and then anticoagulated for preservation $[0.6 \mathrm{ml}$ blood was anticoagulated with ethylene diamine tetraacetic acid (EDTA) for the detection of Treg cells using flow cytometry; $0.9 \mathrm{ml}$ blood was anticoagulated with heparin sodium for the detection of Th17 cells using flow cytometry and the detection of IL-17 content using enzyme-linked immunosorbent assay (ELISA)]. After neurological severity scoring, the rats were euthanized by intraperitoneal injection of $\geq 100 \mathrm{mg} / \mathrm{kg}$ pentobarbital sodium, and their brains were immediately removed. From each group, six brain tissues were used to detect protein and gene expression in tissue homogenates, six brain tissues were embedded in paraffin sections for histopathological observation, and six brain tissues were used for brain water content testing.

Neurological severity assessment. The neurological functioning of the rats was studied using the modified neurological severity score (18). It comprised a combination of motor, sensory, and reflex tests. The score ranged from 0 to 18 , according to the severity of injury. The higher score indicated the most severe injury ( 0 , no injury; $1-6$, mild injury; $7-12$, average moderate injury; 13-18, severe injury; 18 , maximum injury). Neurological function was assessed one day after TBI by an observer who was blinded to the treatment groups. Scoring criteria are shown in Table SI.

Brain water content. The injured brain tissues of rats with TBI were collected and weighed on an electronic balance to obtain the wet weight. The brain tissues were then dried in an oven at $85^{\circ} \mathrm{C}$ for $24 \mathrm{~h}$ and the dry weight was obtained. Brain water content (\%) was calculated as; (wet weight-dry weight)/wet weight $\mathrm{x} 100$.

Histological staining. Brain tissues were fixed, dehydrated, and embedded in paraffin: The brain tissues were fixed with $4 \%$ paraformaldehyde at room temperature for $24 \mathrm{~h}$, then dehydrated with gradient ethanol, soaked in xylene for $30 \mathrm{~min}$, embedded in paraffin, and sliced into tissue sections (5 $\mu \mathrm{m})$. Next, the paraffin sections were deparaffinized and hydrated. The sections were then stained with hematoxylin and eosin (H\&E) for $5 \mathrm{~min}$ and differentiated in ethanol with $0.6 \%$ hydrochloric acid for $30 \mathrm{sec}$, followed by counterstaining with acidified eosin alcohol ( $\mathrm{pH} 4.2$ ) for $3 \mathrm{~min}$. Subsequently, the tissues were dehydrated and cleared. The pathological areas of the brain were observed under an optical microscope (Olympus Optical Co., Ltd.).

Terminal deoxynucleotidyl transferase (TdT)-mediated dUTP nick end-labeling (TUNEL) staining was performed on the sections of the rats' brain tissues according to the manufacturer's instructions (Thermo Fisher). The sections were fixed in ethanol and acetic acid (2:1), washed with phosphate-buffered saline (PBS), and then incubated with protease $\mathrm{K}(100 \mu \mathrm{g} / \mathrm{ml})$ at room temperature for 15-30 min. Subsequently, the sections were washed with PBS, cultured in the presence of $3 \%$ hydrogen peroxide, and permeabilized with $0.5 \%$ Triton $\mathrm{X}-100$. After rewashing, the sections were incubated with the TUNEL reaction mixture at $37^{\circ} \mathrm{C}$ for $1 \mathrm{~h}$. Finally, the sections were developed with $0.03 \%$ 2,4-diaminobutyric acid (DAB), counterstained with hematoxylin, and 
observed under a fuorescence microscope (Olympus IX71; Olympus Corporation) after sealing.

The brain tissue sections were incubated with the primary antibodies (rabbit anti-rat IL-17A polyclonal antibody, 1:500, ab214588, Abcam; rabbit TNF- $\alpha$ polyclonal antibody, 1:50, PA1-40281, Invitrogen-Thermo Fisher Scientific; rabbit IL-1 $\beta$ polyclonal antibody, 1:400, BS-6319R, Invitrogen) at $4^{\circ} \mathrm{C}$ overnight and then incubated with the secondary antibody (goat anti-rabbit IgG H\&L (horseradish peroxidase), 1:2,000, ab205718; Abcam) at $37^{\circ} \mathrm{C}$ for $2 \mathrm{~h}$. After washing with PBS, the sections were developed with $\mathrm{DAB}$ as the substrate and $\mathrm{H}_{2} \mathrm{O}_{2}$ as the catalyst. The sections were observed and analyzed under a microscope after sealing.

Flow cytometry. Treg cells in the peripheral blood were detected according to the manufacturer's instructions $(19,20)$. Peripheral venous blood and cluster for differentiation of antibodies, including CD4 (1:250, ab59474; Abcam), CD25 (1:250, ab210330; Abcam), and forkhead box P3 (Foxp3; 1:250, ab210232; Abcam) were added to the detection tube, with immunoglobulin G (IgG) (1:250, ab205718; Abcam) acting as the isotype control. The tube was then placed in the dark at $4^{\circ} \mathrm{C}$, followed by detection using a flow cytometer, and subsequently, Th17 cells in the peripheral blood were detected. Peripheral blood mononuclear cells (PBMCs) were also isolated. Subsequently, $1 \mathrm{ml}$ cell suspension, $50 \mathrm{ng}$ phorbol 12-myristate 13 -acetate (PMA), $1 \mu \mathrm{g}$ ionomycin, and $0.7 \mu 1$ monensin were added into each well of a 6 -well plate and then cultured at $37^{\circ} \mathrm{C}$ for $6 \mathrm{~h}$. After $5 \mathrm{~min}$ of centrifugation at room temperature and $200 \mathrm{x} \mathrm{g}$, the cells were transferred to a flow detection tube, resuspended in PBS, incubated with CD3 (1:1,000, ab16669; Abcam) and CD8 antibodies (1:1,000, ab101500; Abcam), and supplemented with a fixed membrane-penetrating agent. After washing and resuspending, the cells were incubated with antibody IL-17A (1:250, ab193955; Abcam) with IgG acting as the isotype control, and then the cells were placed in the dark, followed by detection using a flow cytometer.

The cells were collected and resuspended at $1 \times 10^{6}$ cells $/ \mathrm{ml}$. Annexin V-FITC and propidium iodide (PI) (5 $\mu \mathrm{l})$ were added into each tube, which were then incubated in the dark at room temperature for $10 \mathrm{~min}$. Thereafter, the stained cells were analyzed by flow cytometry.

ELISA. The inflammatory factor levels of IL-17, tumor necrosis factor- $\alpha$ (TNF- $\alpha)$, and IL- $1 \beta$ in the brain tissues or cells after the different treatments were measured based on the enzyme-linked immunosorbent assay (ELISA) kit (R\&D Systems) instructions.

Reverse transcriptase-quantitative polymerase chain reaction. Total RNA was extracted from brain tissue using a TRIzol ${ }^{\circledR}$ reagent (Invitrogen). The purity of the extracted high-quality RNA was measured by ultraviolet analysis and formaldehyde deformation electrophoresis. Reverse transcriptase-quantitative polymerase chain reaction (RT-qPCR) was performed using an RT-qPCR kit (Thermo Fisher Scientific). Primers (Table I) were designed and synthesized by Sangon Biotech Co., Ltd. (Shanghai). Glyceraldehyde-3-phosphate dehydrogenase (GAPDH) served as an internal reference. Amplification and dissolution curves were confirmed after the
Table I. Primer sequences for RT-qPCR.

\begin{tabular}{ll}
\hline Gene name & \multicolumn{1}{c}{ Primer sequence } \\
\hline U6 & F: 5'-CGCTTCGGCAGCACATATAC-3' \\
& R: 5'-AATATGGAACGCTTCACGA-3' \\
$m i R-145-3 p$ & F: 5'-GGATTCCTGGAAATACTGT-3' \\
& R: 5'-AGAACAGTATTTCCAGGAATC-3' \\
$m i R-28-3 p$ & F: 5'-CACTAGATTGTGAGCTCCT-3' \\
& R: 5'-TCCAGGAGCTCACAATCTAG-3' \\
$m i R-532-5 p$ & F: 5'-CATGCCTTGAGTGTAGGACC-3' \\
& R: 5'-CGGTCCTACACTCAAGGCATG-3' \\
$m i R-29 b-3 p$ & F: 5'-TAGCACCATTTGAAATCAGTG-3' \\
& R: 5'-AACACTGATTTCAAATGGTGC-3' \\
GAPDH & F: 5'-GGGAGCCAAAAGGGTCAT-3' \\
& R: 5'-GAGTCCTTCCACGATACCAA-3' \\
NFATC2 & F: 5'-ATGCAGAGAGAGGCTGCGTTCAG-3' \\
& R: 5'-TCATAATATGTTTTGTATCCAGC-3'
\end{tabular}

F, forward; R, reverse; NFATc2, nuclear factor of activated T cells c2; GAPDH, glyceraldehyde-3-phosphate dehydrogenase.

reaction. The relative expression of genes was calculated by the $2^{-\Delta \Delta \mathrm{Cq}}$ method (21).

Western blot analysis. The brain tissues were added with pre-cooled Tris- $\mathrm{HCl}$ buffer $(\mathrm{pH} 7.4)$ at the ratio of 1:3, and made into brain tissue homogenate with a glass homogenizer in an ice bath, and then the cells or tissue homogenates were lysed in cold RIPA containing a protease inhibitor cocktail [50 mM Tris (pH 7.4), $150 \mathrm{mM} \mathrm{NaCl}, 1 \%$ NP-40, 0.1\% SDS, $1 \mathrm{mM}$ PMSF, $1 \mathrm{mM} \mathrm{NaF}, 1 \mathrm{mM} \mathrm{Na} \mathrm{VO}_{4}, 1 \%$ protease inhibitor cocktail from Sigma, which was added right before use], for $30 \mathrm{~min}$. The lysate was then centrifuged at $4^{\circ} \mathrm{C}$ for $20 \mathrm{~min}$ at $16,000 \mathrm{x} g$ to collect the supernatant. The protein concentration was determined using the Pierce bicinchoninic acid assay kit (Beyotime Biotechnology Co., Ltd.). Equal amounts of protein (20 $\mu \mathrm{l} /$ well) were separated by $7.5 \%$ SDS-polyacrylamide gel electrophoresis and transferred onto polyvinylidene difluoride membranes. The membranes were blocked with 5\% skimmed milk for $1 \mathrm{~h}$ and incubated overnight at $4^{\circ} \mathrm{C}$ with primary antibodies as follows: Recombinant nuclear factor of activated T cells c2 (NFATc2) (1:1,000, ab2722; Abcam), p65 (1:1,000, ab16502; Abcam), p-p65 (1:2,000, ab86299; Abcam), IкB $\alpha$ (1:1,000, ab32518; Abcam), and p-IкB $\alpha$ (1:10,000, ab133462; Abcam). The membranes were then incubated with secondary antibody IgG (1:2,000, ab205718; Abcam). The gray value of the target band was analyzed using ImageJ software (Rawak Software, Inc.) with $\beta$-actin as the internal reference.

Bioinformatics analysis. The target binding bite between miR-145-3p and NFATc2 was analyzed and predicted through Targetscan (http://www.targetscan.org/) (22).

Dual-luciferase reporter gene assay. The NFATc2 fragment containing the miR-145-3p binding site was cloned 
into the pmirGLO vector (Promega). Subsequently, pmirGLO-NFATc2-wild-type (NFATc2-WT) and pmirGLO-NFATc2-mutant-type (NFATc2-MUT) plasmids were constructed. The constructed vectors were transfected into $293 \mathrm{~T}$ cells, and then the cells were transfected with miR-145-3p mimic or miR-NC. Luciferase activity was detected $48 \mathrm{~h}$ after transfection using the dual-luciferase reporter gene assay system (Promega), and the relative activity was calculated as the ratio of firefly luciferase activity to that of Renilla luciferase activity.

Statistical analysis. Data analysis was performed using SPSS 21.0 (IBM Corp.) and GraphPad Prism 8.0 (GraphPad Software Inc.). The Shapiro-Wilk test confirmed that the data were normally distributed. Data are expressed as mean \pm standard deviation. The unpaired t-test was performed to compare analyses between the two groups. One-way analysis of variance (ANOVA) or two-way ANOVA was performed for comparisons among multiple groups, and Tukey's multiple comparison test was used as the post hoc test after ANOVA. The P-value was obtained from the two-tailed test and $\mathrm{P}<0.05$ indicated statistical significance.

\section{Results}

Propofol reduced brain injury in TBI rats, inhibited IL-17 expression, and maintained Th17/Treg balance. The neurological score of the rats in the TBI+P group was significantly lower than that in the TBI group (Fig. 1A). The brain water content of rats after TBI was increased markedly, and propofol decreased the brain water content (Fig. 1B). The results of H\&E and TUNEL staining showed that propofol notably relieved brain injury and reduced the neuronal apoptosis rate (Fig. 1C). IL-17 expression in rat peripheral blood was detected using ELISA, and the ratio of Th17/Treg cells was measured using flow cytometry. Compared with the TBI group, the TBI+P group had significantly reduced IL-17 expression (Fig. 1D), Th17/Treg cell ratio (Fig. 1E) and inflammation in the rats' brains (Fig. 1F) (all $\mathrm{P}<0.05$ ).

Propofol upregulated miR-145-3p expression in the brain of rats with $T B I$. It has been reported that miR is differentially expressed in the plasma of rats with TBI, and miR-145-3p expression is decreased after TBI (23). We used RT-qPCR to verify some differentially expressed miRs mentioned in the literature and found that the expression of miR-145-3p was significantly reduced after TBI (Fig. 2A), which has been reported to affect the response of Th17 cells (24). Our study demonstrated that propofol upregulated miR-145-3p expression in the brain of rats with TBI (Fig. 2B) (all $\mathrm{P}<0.05$ ). Therefore, we focused on miR-145-3p.

Inhibition of miR-145-3p reversed the effect of propofol on brain injury. To verify that the effect of propofol on rats with TBI was realized through the upregulation of miR-145-3p, we injected ant-145 into the lateral ventricles of the rats (Fig. 3A). Subsequently, we found that the inhibition of miR-145-3p reversed the ameliorative effect of propofol on brain injury. The neurological score, brain water content, neuronal apoptosis rate, IL-17 expression in peripheral blood, Th17/Treg cell ratio, and inflammatory factor-positive cell rates were significantly higher in the TBI+P+ant-145 group than in the TBI+P group (Fig. 3B-G) $($ all $\mathrm{P}<0.05)$. This indicated that the inhibition of miR-145-3p reversed the effect of propofol on brain injury.

miR-145-3p inhibited NFATc2 expression. Through analysis and prediction using the bioinformatics website (http://www. targetscan.org/), we found that miR-145-3p targeted NFATc2 (Fig. 4A) (22), and the dual-luciferase reporter gene assay confirmed this targeted relationship (Fig. 4B). NFATc2 expression in the brain tissues of rats in different treatment groups was detected using RT-qPCR and western blot analysis. The results demonstrated that compared with those in the sham group, the rat brain tissues in the TBI group showed increased NFATc2 mRNA expression and protein level; the upregulation of miR-145-3p induced by propofol reduced the NFATc2 mRNA expression and protein level, while the inhibition of miR-145-3p partially reversed NFATc2 mRNA expression and protein level (Fig. 4C and D) (all $\mathrm{P}<0.05$ ). These results suggested that miR-145-3p negatively regulated the expression of NFATc2.

Propofol inhibited brain inflammation in rats with TBI via the

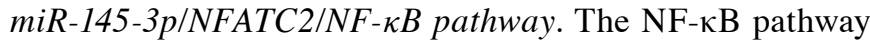
is a classic inflammation pathway. We observed the inhibitory effect of propofol on inflammation in rats with TBI and found that propofol exerted therapeutic effects by upregulating miR-145-3p expression. We speculated that the downstream effects of miR-145-3p may be associated with the NF- $\kappa B$ pathway. Phosphorylation levels of NF-kB pathway-related proteins (p-p65 and $\mathrm{p}-\mathrm{I \kappa} \mathrm{B} \alpha$ ) were detected and found to be significantly increased in rats with TBI compared with sham rats. Propofol inhibited the phosphorylation levels of these proteins, while inhibition of miR-145-3p notably increased their phosphorylation levels (Fig. 5) (all $\mathrm{P}<0.01$ ). It was suggested that propofol inhibited brain inflammation in rats with TBI via the miR-145-3p/NFATC2/NF-kB pathway.

\section{Discussion}

After the primary mechanical insult, TBI causes secondary injury through the activation of pro-inflammatory factors, loss of neuronal and cerebral edema, resulting in neurological dysfunction (25). Inflammation has been demonstrated to play a vital role in the progression of secondary brain injury (26). Propofol is a clinical anesthetic commonly used to treat patients with TBI (27). Although propofol has been reported to inhibit the inflammatory response (28), research on the underlying mechanism of propofol in TBI has been less encouraging. In this study, we demonstrated that propofol inhibited brain inflammation in rats with TBI via the miR-145-3p/NFATc2/NF- $\mathrm{BB}$ axis, thereby alleviating brain injury in rats with TBI.

First, the TBI model was established in rats. The neuroinflammatory response after TBI represents a critical secondary injury factor that can drive sustained neuronal damage (29). IL-17 is an inflammatory mediator that influences the pathogenesis of brain injury $(30,31)$. We found that the neurological score, brain edema, and neuronal apoptosis rate of the propofol-treated rats with TBI were significantly 

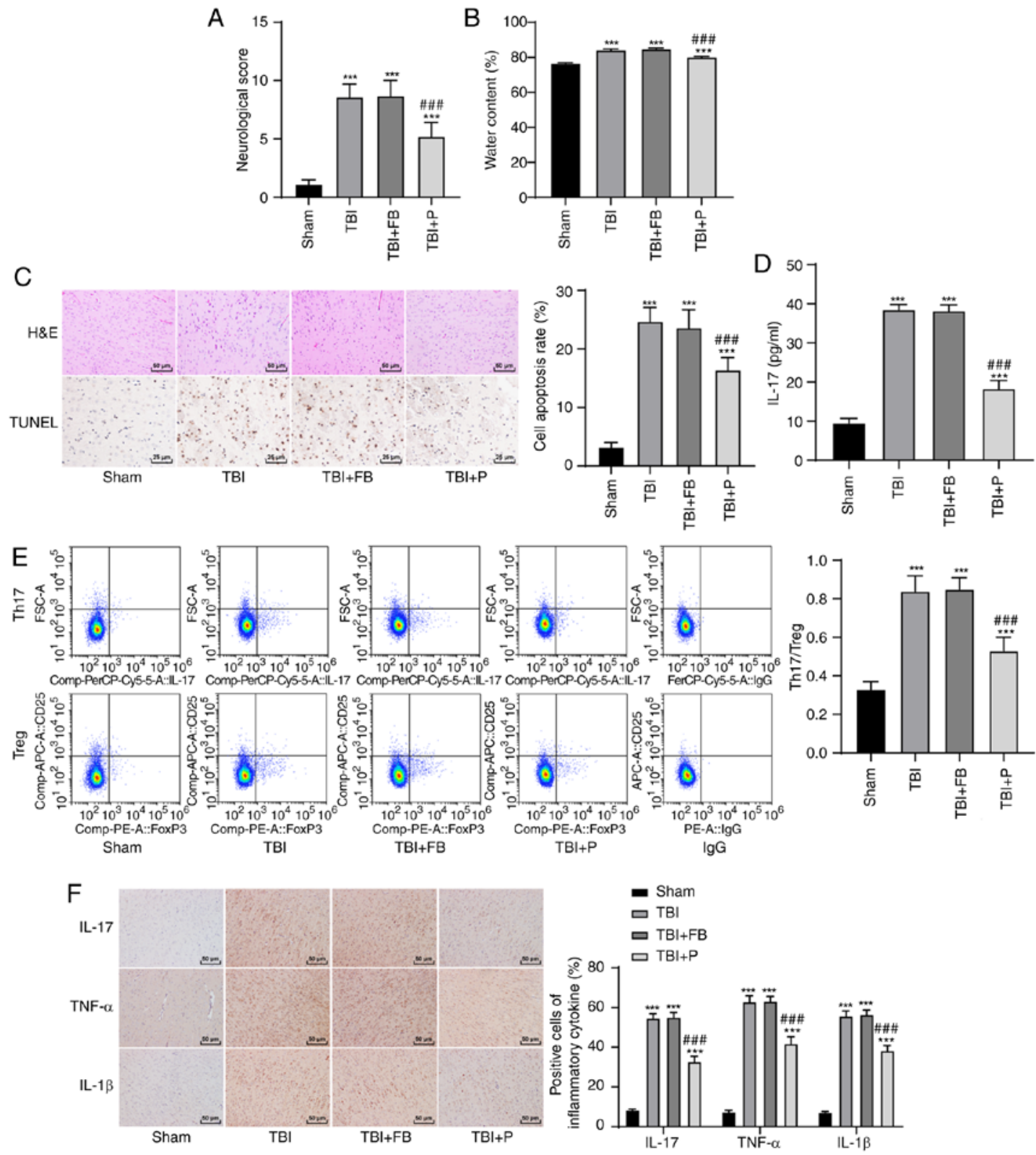

Figure 1. Propofol reduced brain injury in rats with TBI, inhibited IL-17 expression, and maintained Th17/Treg balance. (A) Neurological score, n=18; (B) brain water content, $\mathrm{n}=6$; (C) brain tissue sections of rats with TBI were stained with H\&E and TUNEL, and the apoptotic rate was expressed using the TUNEL-positive cell rate, $n=6$; (D) IL-17 expression in the rats' peripheral blood was detected using ELISA, $n=18$; (E) the ratio of Th17/Treg cells in the rats peripheral blood was detected by flow cytometry, $n=18$; (F) the cells that were positive for inflammatory factors in the rats' brains were detected using immunohistochemistry, $\mathrm{n}=6$. Each experiment was repeated three times. Data were analyzed using one-way ANOVA, followed by Tukey's multiple comparisons test. ${ }^{* * * *}$ Compared with the sham group, $\mathrm{P}<0.001 ;{ }^{* \# \#}$ Compared with the TBI group, $\mathrm{P}<0.001$.
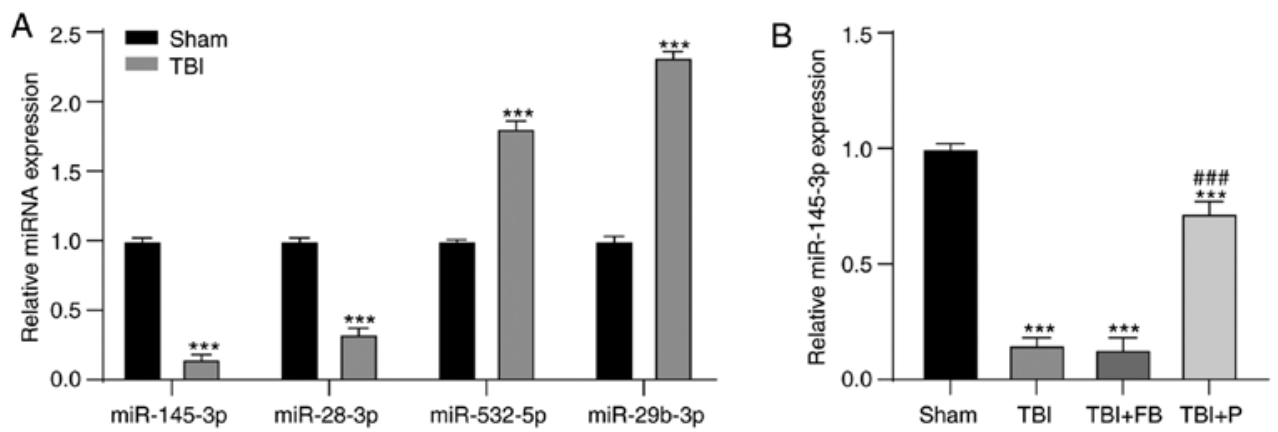

Figure 2. Propofol upregulated miR-145-3p expression in the brain of rats with TBI. (A-B) Expressions of miRs in the brain tissues of rats were detected using RT-qPCR. (A) Five miRs differentially expressed in the literature as validation; (B) expression of miR-145-3p under different treatments, $n=6$. Each experiment was repeated three times. Data were analyzed using one-way ANOVA, followed by Tukey's multiple comparisons test. ${ }^{* * *}$ Compared with the sham group, $\mathrm{P}<0.001 ;{ }^{\# \#}$ Compared with the TBI group, $\mathrm{P}<0.001$. 
A

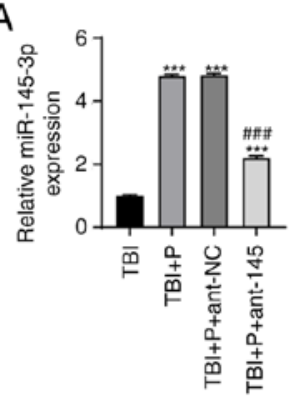

D

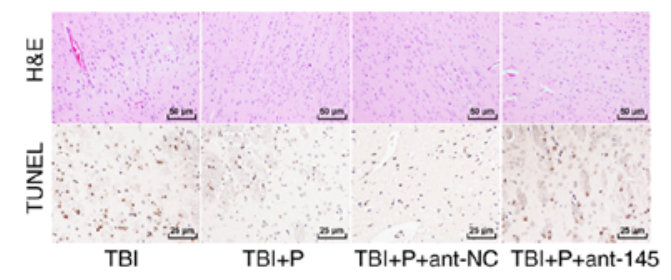

B

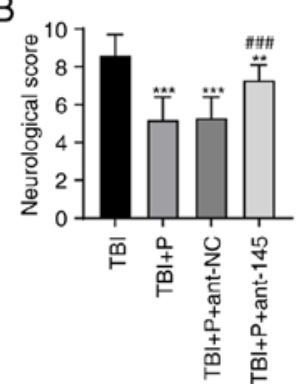

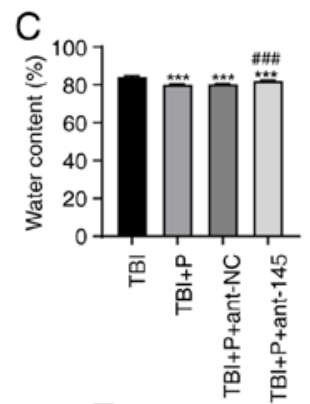

E
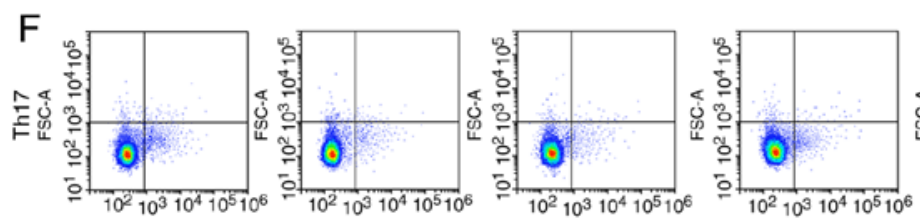

Comp-PerCP-Cy5-5-A-
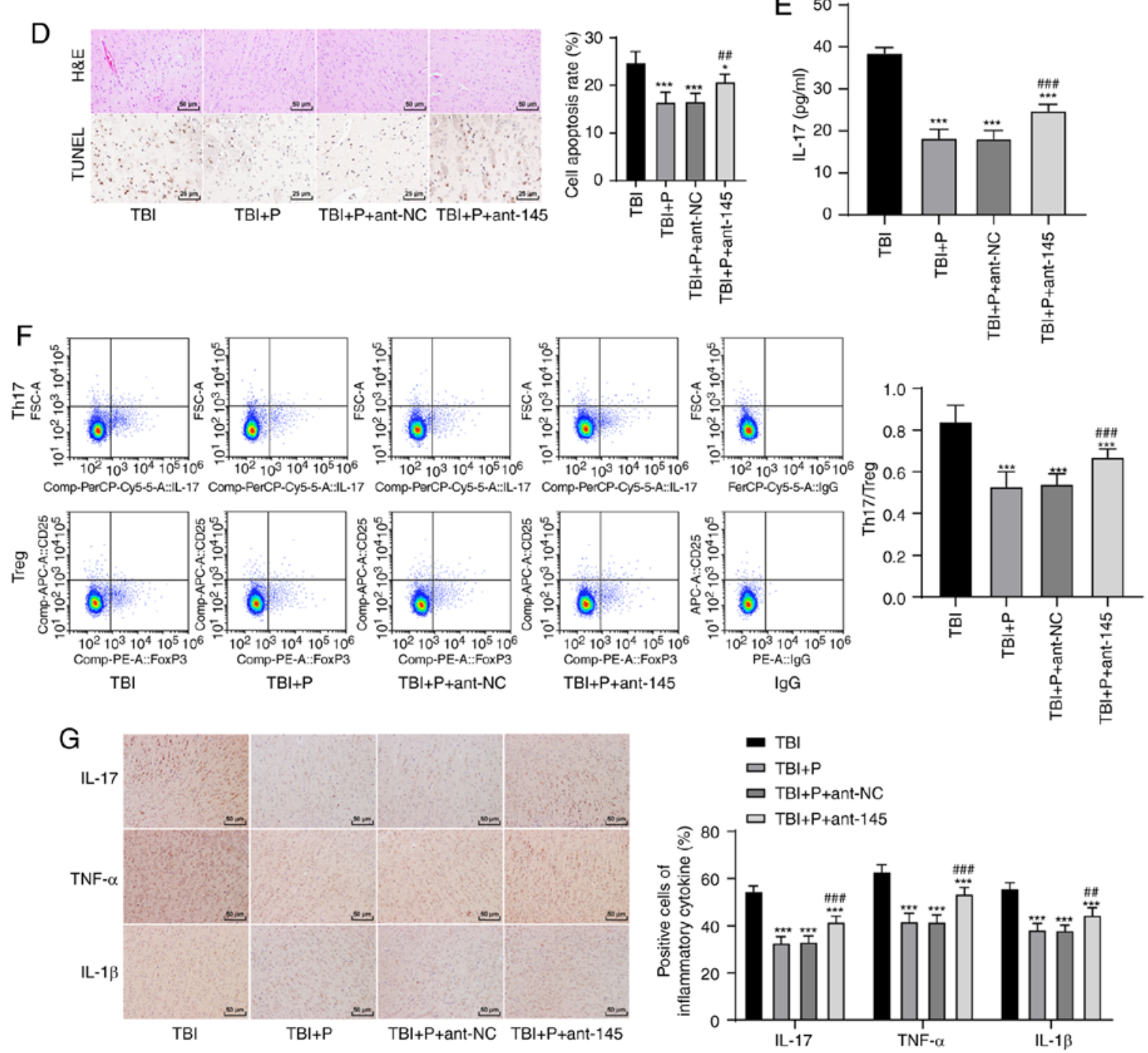

Figure 3. Inhibition of miR-145-3p reversed the effect of propofol on brain injury. (A) Expression of miR-145-3p in brain tissues of rats after injection of antagomiR into lateral ventricle was detected using RT-qPCR; (B) neurological score, $\mathrm{n}=18$; (C) brain water content, $\mathrm{n}=6$; (D) brain tissue sections of rats with TBI were stained with H\&E and TUNEL, and the apoptotic rate was expressed using the TUNEL-positive cell rate, $\mathrm{n}=6$; (E) IL-17 expression in the rats peripheral blood was detected using ELISA, $n=18 ;(F)$ the ratio of Th17/Treg cells in the rats' peripheral blood was detected by flow cytometry, $n=18 ;(\mathrm{G})$ the cells that were positive for inflammatory factors in the rats' brains were detected using immunohistochemistry, $\mathrm{n}=6$. Each experiment was repeated three times. Data were analyzed using one-way ANOVA, followed by Tukey's multiple comparisons test. ${ }^{*} \mathrm{P}<0.05,{ }^{* *} \mathrm{P}<0.01,{ }^{* * *} \mathrm{P}<0.001$, compared with the TBI group; ${ }^{\#} \mathrm{P}<0.01,{ }^{\# \#} \mathrm{P}<0.001$, compared with the TBI+P group.

decreased, indicating that propofol could relieve brain injury in rats with TBI. In addition, Th17 cells contribute to autoimmunity and inflammation, while Treg cells maintain immune homeostasis (32). An imbalance of Th17/Treg cells is therefore also associated with autoimmune and inflammatory diseases (33). To the best of our knowledge, this is the first study to demonstrate that propofol inhibits IL-17 expression in rats with TBI and maintains the balance of Th17/Treg cells. It is worth noting that the expression of some miRs in the cerebral cortex and hippocampus of rats with TBI was abnormal, which could accelerate or suppress secondary brain injury (34). Previous findings demonstrated that propofol attenuates hypoxia-induced brain injury by upregulating miR-137 expression (35). miR-145-3p is widely acknowledged to be a tumor suppressor (36); however, relatively little is known about the role of miR-145-3p in TBI. In this study, we 


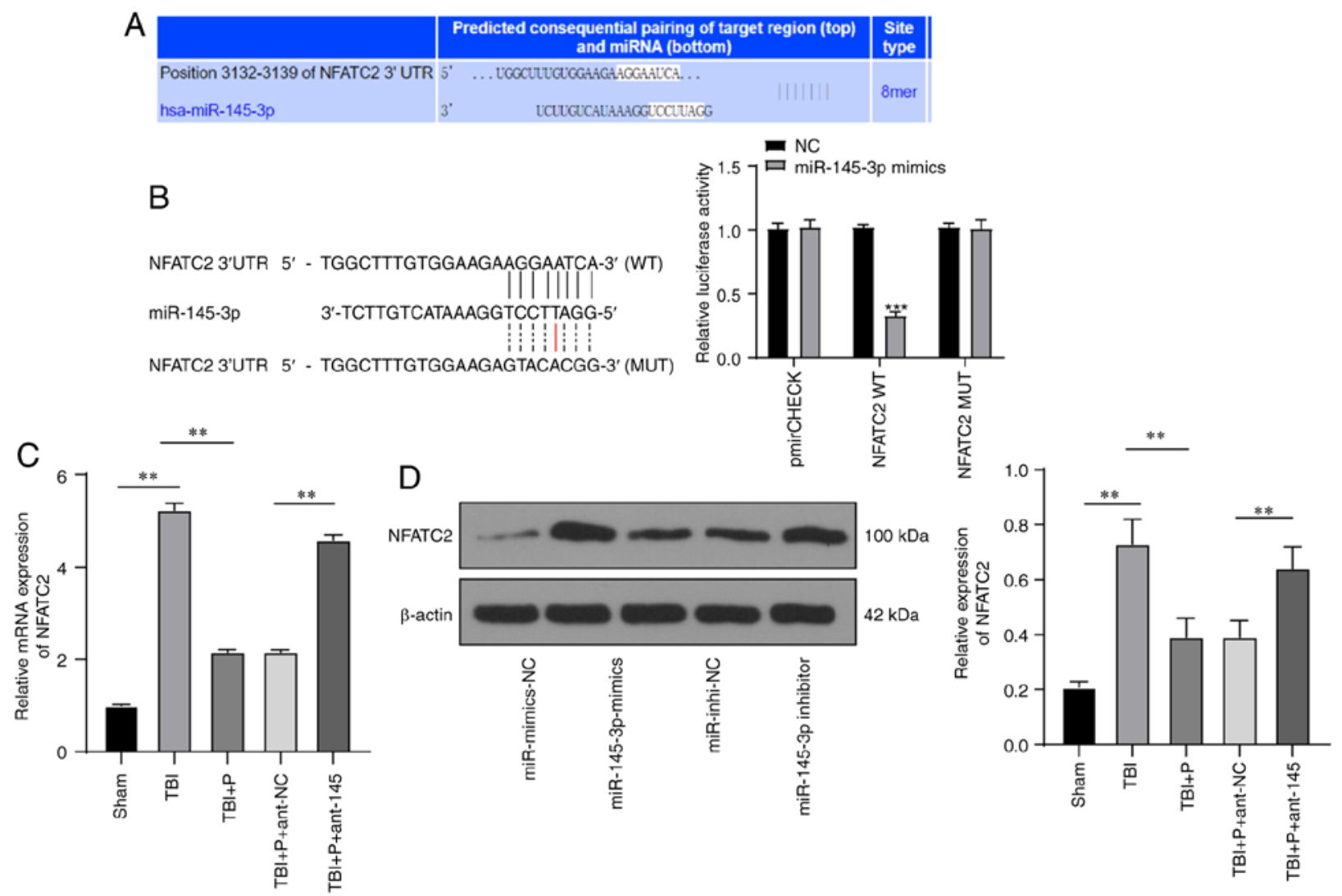

Figure 4. miR-145-3p inhibited the expression of NFATc2. (A) Analysis and prediction of binding site between miR-145-3p and NFATc2 online (http://www. targetscan.org/); (B) the targeting relationship between miR-145-3p and NFATc2 was confirmed using the dual-luciferase reporter gene assay; (C and D) NFATc2 expression in brain tissues of rats in each treatment group was detected using RT-qPCR and western blotting. Each experiment was repeated three times. Data were analyzed using one-way ANOVA, followed by Tukey's multiple comparisons test. ${ }^{* *} \mathrm{P}<0.01,{ }^{* * * *} \mathrm{P}<0.001$, compared with each $\mathrm{NC}$ group.
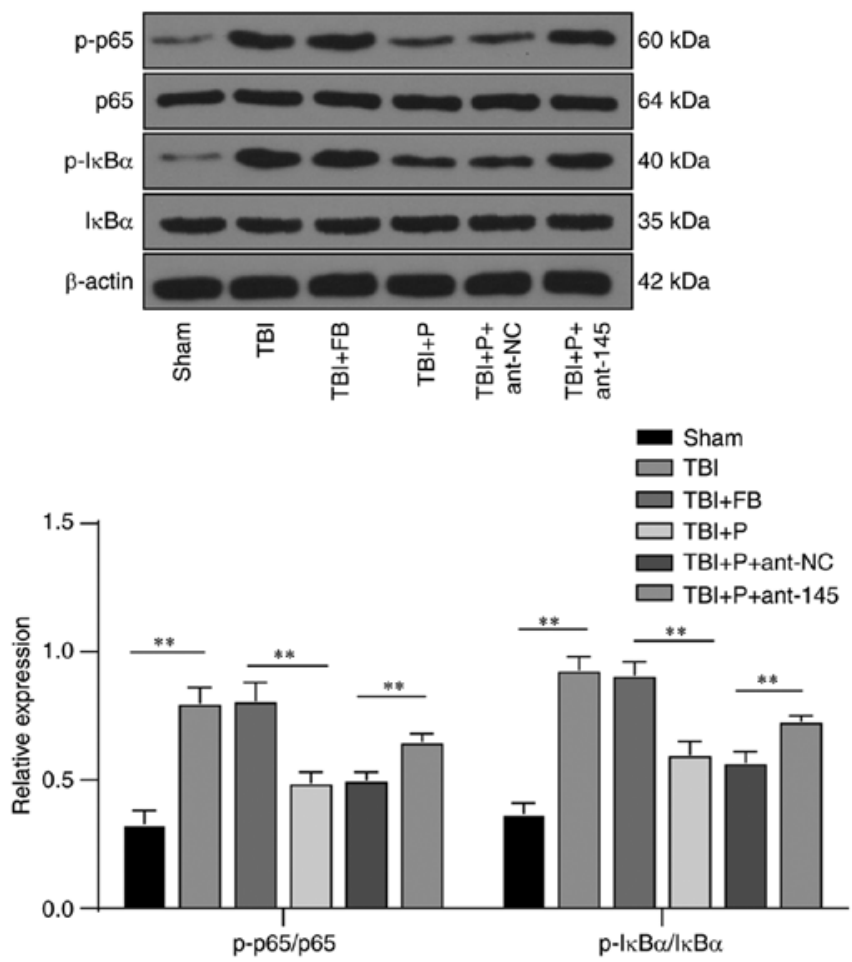

Figure 5. Propofol inhibited phosphorylation of NF- $\kappa B$ pathway-related proteins. Phosphorylation and protein level of NF- $\kappa B$ pathway-related proteins in brain tissues of rats with TBI under different treatments were detected using western blotting, $n=6$. Data were analyzed using one-way ANOVA, followed by Tukey's multiple comparisons test. ${ }^{* *} \mathrm{P}<0.01$. found that miR-145-3p expression was notably decreased in rats with TBI and increased after propofol treatment. Many miRs have been found to be differentially expressed in rats with TBI, including miR-145-3p (23). In this study, the inhibition of miR-145-3p reversed the effect of propofol on brain injury, which confirmed that the upregulation of miR-145-3p is a vital part of propofol's protective effect on rats with TBI. Overexpression of miR-145 has been demonstrated to ameliorate astrocyte injury in cerebral ischemic stroke by targeting aquaporin 4 (37). Wang et al have also shown that lncRNA MALAT1 silencing protects against cerebral ischemia-reperfusion injury by upregulating miR-145 expression (38).

The miR-145-3p target gene was then explored. NFAT is a carcinogenic transcription factor that is induced under inflammatory conditions (39). NFATc2, a member of the NFAT family, promotes the generation of inflammatory cytokines (40). Of the various miR-149-5p target genes proposed by the bioinformatics website used, we focused on NFATc2. Gerlach et al revealed the key role of NFATc2 in the initiation of inflammation-related colorectal tumors (41). In this study, the targeting relationship between miR-145-3p and NFATc2 was verified using a dual-luciferase reporter gene assay. The upregulation of miR-145-3p reduced NFATc2 mRNA and protein levels, suggesting that miR-145-3p reduces the expression of NFATc2. Intriguingly, Furman showed that blocking NFAT signaling was conducive to normalizing hippocampal synaptic function in rats with TBI (42). NFATc has also been reported to be involved in calcineurin-mediated 
ischemic brain injury (43). Subsequently, we investigated the signaling pathways modulated by miR-145-3p/NFATc 2 . $\mathrm{NF}-\kappa \mathrm{B}$ is a central mediator in inflammatory progression, and the activation of $\mathrm{NF}-\kappa \mathrm{B}$ has been deemed a contributor to disease $(44,45)$. Accumulated evidence has shown that suppression of the NF- $\mathrm{NB}$ signaling pathway could alleviate early brain injury $(46,47)$. Consistent with those findings, our data demonstrated that the phosphorylation levels of $\mathrm{NF}-\kappa \mathrm{B}$ pathway-related proteins in rats with TBI were significantly higher, and propofol could inhibit the phosphorylation levels of these proteins.

In summary, our study demonstrated that propofol could maintain Th17/Treg cell balance and attenuate inflammation in rats with TBI by upregulating miR-145-3p expression and inactivating the $\mathrm{NF}-\kappa \mathrm{B}$ pathway. Our results provide essential evidence of the protective mechanism of propofol in brain injury. Another limitation of this study lies in that the mechanism of propofol protecting TBI rats from brain injury through the miR-145-3p/NFATc 2 axis lacks of further cell experimental verification. In future, primary microglia isolated and cultured from SD rats may be used to carry out further cell experiment in vitro, and prospective trials are to be conducted to refine our clinical guidance.

\section{Acknowledgements}

Not applicable.

\section{Funding}

This study was supported by the Science and Technology Planning Project of Guangdong Province, China (2012B031800161), the Medical Scientific Research Foundation of Guangdong Province, China (A2016573), the Natural Science Foundation of Guangdong Province, China (2018A0303130236), the Natural Science Foundation of Guangdong Province, China (2018A0303130297); the Science and Technology Program of Guangzhou, China (201904010080), the Project of Administration of Traditional Chinese Medicine of Guangdong Province, China (20191006), the Foundation for Basic and Applied Basic Research of Guangdong Province, China (2019A1515110063), and the Medical Scientific Research Foundation of Guangdong Province, China (A2020038).

\section{Availability of data and materials}

The datasets used and/or analyzed during the current study are available from the corresponding author on reasonable request.

\section{Authors' contributions}

$\mathrm{CC}$ and DZ completed the experimental design and were the major contributors in writing the manuscript. $\mathrm{KS}, \mathrm{HL}, \mathrm{JH}$ and LX participated in the design and operation of the experiments. GL, YG, and JH were responsible for literature research and data collection. JC, GL and LN performed the statistical analysis. YC, YG and DY completed the data interpretation and revised the manuscript. WY, PW, and YS ensured the manuscript preparation and the integrity of project management and confirmed the authenticity of all original data. All authors read and approved the final manuscript.

\section{Ethics approval and consent to participate}

This study was performed with the approval of the Medical Research of Guangdong Provincial People's Hospital (Guangdong Academy of Medical Sciences), GDREC2012116H (R1), January 15, 2016. All procedures were strictly performed according to the code of ethics. Great efforts were made to minimize pain in experimental animals.

\section{Patient consent for publication}

Not applicable.

\section{Competing interests}

All authors declare that they have no competing interests.

\section{References}

1. Gardner AJ and Zafonte R: Neuroepidemiology of traumatic brain injury. Handb Clin Neurol 138: 207-223, 2016.

2. Georgiou AP and Manara AR: Role of therapeutic hypothermia in improving outcome after traumatic brain injury: A systematic review. Br J Anaesth 110: 357-367, 2013.

3. Ziebell JM and Morganti-Kossmann MC: Involvement of pro- and anti-inflammatory cytokines and chemokines in the pathophysiology of traumatic brain injury. Neurotherapeutics 7: 22-30, 2010.

4. Boer C, Franschman G and Loer SA: Prehospital management of severe traumatic brain injury: Concepts and ongoing controversies. Curr Opin Anaesthesiol 25: 556-562, 2012.

5. Si L, Wang H and Wang L: Suppression of miR-193a alleviates neuroinflammation and improves neurological function recovery after traumatic brain injury (TBI) in mice. Biochem Biophys Res Commun 523: 527-534, 2020.

6. Helmy A, Carpenter KL, Menon DK, Pickard JD and Hutchinson PJ: The cytokine response to human traumatic brain injury: Temporal profiles and evidence for cerebral parenchymal production. J Cereb Blood Flow Metab 31: 658-670, 2011.

7. Li T, Zhang YM, Han D, Hua R, Guo BN, Hu SQ, Yan XL and $\mathrm{Xu}$ T: Involvement of IL-17 in secondary brain injury after a traumatic brain injury in rats. Neuromolecular Med 19: 541-554, 2017.

8. Luo T, Ji WJ, Yuan F, Guo ZZ, Li YX, Dong Y, Ma YQ, Zhou X and Li YM: Th17/Treg imbalance induced by dietary salt variation indicates inflammation of target organs in humans. Sci Rep 6: 26767, 2016.

9. Shen Y, Tang XY, Yang YC, Ke X, Kou W, Pan CK and Hong SL: Impaired balance of Th17/Treg in patients with nasal polyposis. Scand J Immunol 74: 176-185, 2011.

10. Fulton B and Sorkin EM: Propofol. An overview of its pharmacology and a review of its clinical efficacy in intensive care sedation. Drugs 50: 636-657, 1995.

11. Marik PE: Propofol: An immunomodulating agent. Pharmacotherapy 25: 28S-33S, 2005.

12. Zheng X,Huang H,Liu J,Li M,Liu M and Luo T: Propofol attenuates inflammatory response in LPS-activated microglia by regulating the miR-155/SOCS1 pathway. Inflammation 41: 11-19, 2018.

13. Yu S, Xin W, Jiang Q and Li A: Propofol exerts neuroprotective functions by down-regulating microRNA-19a in glutamic acid-induced PC12 cells. Biofactors 46: 934-942, 2020.

14. Shioya M, Obayashi S, Tabunoki H, Arima K, Saito Y, Ishida T and Satoh J: Aberrant microRNA expression in the brains of neurodegenerative diseases: miR-29a decreased in Alzheimer disease brains targets neurone navigator 3. Neuropathol Appl Neurobiol 36: 320-330, 2010

15. Di Pietro V, Yakoub KM, Scarpa U, Di Pietro C and Belli A: MicroRNA signature of traumatic brain injury: From the biomarker discovery to the point-of-care. Front Neurol 9: 429, 2018. 
16. Henry RJ, Doran SJ, Barrett JP, Meadows VE, Sabirzhanov B, Stoica BA, Loane DJ and Faden AI: Inhibition of miR-155 limits neuroinflammation and improves functional recovery after experimental traumatic brain injury in mice. Neurotherapeutics 16 : 216-230, 2019

17. Li Z, Wang Y, Zeng G, Zheng X, Wang W, Ling Y, Tang H and Zhang J: Increased miR-155 and heme oxygenase-1 expression is involved in the protective effects of formononetin in traumatic brain injury in rats. Am J Transl Res 9: 5653-5661, 2017.

18. Chen J, Li Y, Wang L, Zhang Z, Lu D, Lu M and Chopp M: Therapeutic benefit of intravenous administration of bone marrow stromal cells after cerebral ischemia in rats. Stroke 32: 1005-1011, 2001

19. Miyara M, Yoshioka Y, Kitoh A, Shima T, Wing K, Niwa A, Parizot C, Taflin C, Heike T, Valeyre D, et al: Functional delineation and differentiation dynamics of human $\mathrm{CD}^{+} \mathrm{T}$ cells expressing the FoxP3 transcription factor. Immunity 30: 899-911, 2009.

20. Kustrimovic N, Comi C, Magistrelli L, Rasini E, Legnaro M, Bombelli R, Aleksic I, Blandini F, Minafra B, Riboldazzi G, et al: Parkinson's disease patients have a complex phenotypic and functional Th1 bias: Cross-sectional studies of CD4 ${ }^{+}$ Th1/Th2/T17 and Treg in drug-naive and drug-treated patients. J Neuroinflammation 15: 205, 2018

21. Schmittgen TD and Livak KJ: Analyzing real-time PCR data by the comparative C(T) method. Nat Protoc 3: 1101-1108, 2008.

22. Agarwal V, Bell GW, Nam JW and Bartel DP: Predicting effective microRNA target sites in mammalian mRNAs. Elife 4: e05005, 2015.

23. Wang P, Ma H, Zhang Y, Zeng R, Yu J, Liu R, Jin X and Zhao Y: Plasma exosome-derived MicroRNAs as novel biomarkers of traumatic brain injury in rats. Int J Med Sci 17: 437-448, 2020.

24. Wang J, Zheng S, Xin N, Dou C, Fu L, Zhang X, Chen J, Zhang Y, Geng D, Xiao C, et al: Identification of novel MicroRNA signatures linked to experimental autoimmune myasthenia gravis pathogenesis: Down-regulated miR-145 promotes pathogenetic Th17 cell response. J Neuroimmune Pharmacol 8: 1287-1302, 2013.

25. Liu F, Chen MR, Liu J, Zou Y, Wang TY, Zuo YX and Wang TH: Propofol administration improves neurological function associated with inhibition of pro-inflammatory cytokines in adult rats after traumatic brain injury. Neuropeptides 58: 1-6, 2016.

26. Goodman JC, Van M, Gopinath SP and Robertson CS Pro-inflammatory and pro-apoptotic elements of the neuroinflammatory response are activated in traumatic brain injury. Acta Neurochir Suppl 102: 437-439, 2008.

27. Yu Y, Jian MY, Wang YZ and Han RQ: Propofol ameliorates calpain-induced collapsin response mediator protein-2 proteolysis in traumatic brain injury in rats. Chin Med J (Engl) 128 919-927, 2015

28. Shi SS, Zhang HB, Wang CH, Yang WZ, Liang RS, Chen Y and Tu XK: Propofol attenuates early brain injury after subarachnoid hemorrhage in rats. J Mol Neurosci 57: 538-545, 2015.

29. Corrigan F, Mander KA, Leonard AV and Vink R: Neurogenic inflammation after traumatic brain injury and its potentiation of classical inflammation. J Neuroinflammation 13: 264, 2016

30. Kuwabara T, Ishikawa F, Kondo $M$ and Kakiuchi T: The role of IL-17 and related cytokines in inflammatory autoimmune diseases. Mediators Inflamm 2017: 3908061, 2017.

31. Wang DD, Zhao YF, Wang GY, Sun B, Kong QF, Zhao K, Zhang Y, Wang JH, Liu YM, Mu LL, et al: IL-17 potentiates neuronal injury induced by oxygen-glucose deprivation and affects neuronal IL-17 receptor expression. J Neuroimmunol 212: $17-25,2009$.

32. Lee GR: The balance of Th17 versus Treg cells in autoimmunity. Int J Mol Sci 19: 730, 2018.
33. Guo H, He Z, Li M, Wang T and Zhang L: Imbalance of peripheral blood Th17 and Treg responses in children with refractory Mycoplasma pneumoniae pneumonia. J Infect Chemother 22: 162-166, 2016.

34. Pan YB, Sun ZL and Feng DF: The role of MicroRNA in traumatic brain injury. Neuroscience 367: 189-199, 2017.

35. Chang J, Yan X and Zeng Y: Propofol weakens hypoxia-aroused apoptosis and autophagy via elevating microRNA-137 in neurocytes. Exp Mol Pathol 112: 104327, 2020.

36. Mataki H, Seki N, Mizuno K, Kamikawaji K, Kumamoto T, Koshizuka K, Goto Y and Inoue H: Dual-strand tumor-suppressor microRNA-145 (miR-145-5p and miR-145-3p) coordinately targeted MTDH in lung squamous cell carcinoma. Oncotarget 7: 72084-72098, 2016.

37. Zheng L, Cheng W, Wang X, Yang Z, Zhou X and Pan C: Overexpression of MicroRNA-145 ameliorates astrocyte injury by targeting aquaporin 4 in cerebral ischemic stroke. Biomed Res Int 2017: 9530951, 2017

38. Wang H, Zheng X, Jin J, Zheng L, Guan T, Huo Y, Xie S, Wu Y and Chen W: LncRNA MALAT1 silencing protects against cerebral ischemia-reperfusion injury through miR-145 to regulate AQP4. J Biomed Sci 27: 40, 2020.

39. Baumgart S, Chen NM, Zhang JS, Billadeau DD, Gaisina IN, Kozikowski AP, Singh SK, Fink D, Ströbel P, Klindt C, et al: GSK-3 $\beta$ Governs Inflammation-Induced NFATc2 Signaling Hubs to Promote Pancreatic Cancer Progression. Mol Cancer Ther 15: 491-502, 2016.

40. Qi H, Yang Z, Dai C, Wang R, Ke X, Zhang S, Xiang X, Chen K, Li C, Luo J, et al: STAT3 activates MSK1-mediated histone H3 phosphorylation to promote NFAT signaling in gastric carcinogenesis. Oncogenesis 9: 15, 2020.

41. Gerlach K, Daniel C, Lehr HA, Nikolaev A, Gerlach T, Atreya R, Rose-John S, Neurath MF and Weigmann B: Transcription factor NFATc2 controls the emergence of colon cancer associated with IL-6-dependent colitis. Cancer Res 72: 4340-4350, 2012.

42. Furman JL, Sompol P, Kraner SD, Pleiss MM, Putman EJ, Dunkerson J, Mohmmad Abdul H, Roberts KN, Scheff SW and Norris CM: Blockade of astrocytic Calcineurin/NFAT signaling helps to normalize hippocampal synaptic function and plasticity in a rat model of traumatic brain injury. J Neurosci 36: 1502-1515, 2016

43. Zhang YJ, Mei HS, Wang C, Wang YL and Zhang YJ: Involvement of nuclear factor of activated T-cells (NFATc) in calcineurin-mediated ischemic brain damage in vivo. Yao Xue Xue Bao 40: 299-305, 2005.

44. Choi MC, Jo J, Park J, Kang HK and Park Y: NF- $\kappa$ B signaling pathways in osteoarthritic cartilage destruction. Cells 8: 734, 2019.

45. DiDonato JA, Mercurio F and Karin M: NF- $\kappa B$ and the link between inflammation and cancer. Immunol Rev 246: 379-400, 2012.

46. Qu J, Zhao H, Li Q, Pan P, Ma K, Liu X, Feng H and Chen Y: MST1 suppression reduces early brain injury by inhibiting the NF- $\mathrm{B} /$ MMP-9 pathway after subarachnoid hemorrhage in mice. Behav Neurol 2018: 6470957, 2018.

47. Zeng J, Chen Y, Ding R, Feng L, Fu Z, Yang S, Deng X, Xie Z and Zheng S: Isoliquiritigenin alleviates early brain injury after experimental intracerebral hemorrhage via suppressing ROSand/or NF- $\kappa \mathrm{B}$-mediated NLRP3 inflammasome activation by promoting Nrf2 antioxidant pathway. J Neuroinflammation 14: $119,2017$.

(i) $(-)$ This work is licensed under a Creative Commons Attribution-NonCommercial-NoDerivatives 4.0 International (CC BY-NC-ND 4.0) License. 